control group. In addition, Bacteriodes, Cricetibacter, Alistipes, Lachnospira, Dielma, Akkermansia, Sutterella, Anaerofilum, Ruminococcease-UCG007, Acetanaerobacterium; and Copropaacter were lower than the control group. There was no difference between the uveitis, mucocutaneous and vascular involvement groups in terms of alpha (Chao-1 and Shannon) and beta (Bray-Curtis) microbiota diversity and wealth indices $(p>0.05)$ while we obtained a significant $p$ value of the beta diversity between three groups in weighted UniFrac PCoA $(p<0.05)$. When we compared 3 three different system involvement (Eye, Mucocutaneous and Vascular), The LEfSe provides us with cladograms of six-level (from kingdom to genus). We found difference for the genera Lachnospiraceae NK4A136 in uveitis group, Dialister, intestinomonas and Marvinbryantia in mucocutaneous group and Gemella in vascular involvement group.

Conclusion: There was a significant difference in the composition of intestinal microbiota in Behçet's disease compared to healthy adults. We found also found the different clinical forms of Behcet's disease have some different gut microbiota composition. Especially in Behçet's disease, it will be useful to evaluate Catenibacterium, Collinsella and Eggerthella increase, Bacteroides and Akkermansia decrease in larger series. In addition, due to the increase in the Eggerthella lenta strain observed both in the FMF and Behcet patient group, it is useful to make more detailed metagenomic analyzes regarding the role of this agent in the etiopathogenesis and course of rheumatic diseases.

Disclosure of Interests: None declared

DOI: 10.1136/annrheumdis-2020-eular.3626

\section{AB0493 COMPARISON OF EFFICACY AND SAFETY BETWEEN RITUXIMAB AND CYCLOPHOSPHAMIDE IN REMISSION INDUCTION THERAPY FOR JAPANESE ANCA-ASSOCIATED VASCULITIS(AAV) PATIENTS; A SINGLE CENTER RETROSPECTIVE ANALYSIS}

M. Kato ${ }^{1}$, H. Shimada ${ }^{1}$, S. Nakashima ${ }^{1}$, M. Mahmoud Fahmy Mansour ${ }^{1}$, R. Wakiya ${ }^{1}$, T. Miyagi ${ }^{1}$, K. Sugihara ${ }^{1}$, Y. Ushio ${ }^{1}$, T. Kameda ${ }^{1}$, H. Dobashi ${ }^{1}$. ${ }^{1}$ Kagawa University, Division of Hematology, Rheumatology and Respiratory Medicine, Department of Internal Medicine, Kagawa, Japan

Background: Rituximab(RTX) and Cyclophosphamide(CY) has been indicated for ANCA-associated vasculitis(AAV) as remission induction therapy. However, older age and renal disfunction were independent predictor of treatment related adverse effects in remission induction with $C Y$ in recent reports ${ }^{1)}$. Japanese AAV

Table 1. Comparison of clinical characteristics at baseline between RTX and $\mathrm{CY}$ groups.

\begin{tabular}{|c|c|c|c|}
\hline & $\begin{array}{c}\mathrm{RTX} \\
(\mathrm{n}=20)\end{array}$ & $\begin{array}{c}C Y \\
(n=20)\end{array}$ & $p$ value \\
\hline Age, year & $72.3 \pm 11.0$ & $74.8 \pm 8.01$ & 0.55 \\
\hline female, n (\%) & $16(80)$ & $18(90)$ & 0.33 \\
\hline AAV type & & & 0.14 \\
\hline MPA, n (\%) & $13(65)$ & $17(85)$ & \\
\hline GPA, n (\%) & $7(35)$ & $3(15)$ & \\
\hline Newly diagnosis, n (\%) & 7 (35) & $4(20)$ & 0.24 \\
\hline \multicolumn{4}{|l|}{ ANCA positivity } \\
\hline MPO, n (\%) & $20(100)$ & $17(85)$ & 0.12 \\
\hline PR3, n (\%) & $1(5)$ & $2(10)$ & 0.50 \\
\hline negative, n (\%) & $0(0)$ & $2(10)$ & 0.24 \\
\hline eGFR (mL/min) & $55.0 \pm 29.5$ & $58.1 \pm 21.5$ & 0.45 \\
\hline \multicolumn{4}{|l|}{ Organ involvement } \\
\hline General, n (\%) & $14(70)$ & $19(95)$ & $0.046^{*}$ \\
\hline Cutaneous, n (\%) & $1(5)$ & $3(15)$ & 0.30 \\
\hline Eyes, n (\%) & $4(20)$ & $1(5)$ & 0.17 \\
\hline ENT, n (\%) & $5(25)$ & $5(25)$ & 1.00 \\
\hline Pulmonary, n (\%) & $14(70)$ & $15(75)$ & 0.50 \\
\hline Renal, n (\%) & $14(70)$ & $13(65)$ & 0.50 \\
\hline Neurologic, n (\%) & $8(40)$ & $4(20)$ & 0.15 \\
\hline BVAS & $13.8 \pm 6.6$ & $14.1 \pm 7.6$ & 0.73 \\
\hline
\end{tabular}

RTX: Rituximab, CY: Cyclophosphamide, ANCA: antineutrophil cytoplasmic autoantibody, AAV: ANCA-associated vasculitis, GPA: granulomatosis with polyangiitis, MPA: microscopic polyangiitis, ENT: Ear, Nose, and Throat, BVAS Birmingham Vasculitis Activity Score, ${ }^{*} p<0.05$

Table 2. Adverse effects through 12 months. $\%=$ patients with 1 effects

\begin{tabular}{lccc}
\hline & $\begin{array}{c}\mathbf{R T X} \\
(\mathbf{n = 2 0 )}\end{array}$ & $\begin{array}{c}\mathbf{C Y} \\
\mathbf{( n = 2 0 )}\end{array}$ & p value \\
\hline Death, n (\%) & $1(5)$ & $0(0)$ & 0.50 \\
Infections, n (\%) & $7(35)$ & $8(30)$ & 0.46 \\
Serious (grade 3), n (\%) & $4(15)$ & $3(10)$ & 0.50 \\
Malignancy, n (\%) & $1(5)$ & $0(0)$ & 0.50 \\
VTE, n (\%) & $1(5)$ & $0(0)$ & 0.50 \\
AMI, n (\%) & $1(5)$ & $0(0)$ & 0.50 \\
Leukopenia, n (\%) & $3(15)$ & $3(10)$ & 0.70 \\
Necrosis of femoral head, n (\%) & $0(0)$ & $1(5)$ & 0.50 \\
Mediastinal emphysema & $0(0)$ & $1(5)$ & 0.50 \\
\hline
\end{tabular}

VTE: venous thrombotic emboli, AMI: acute myocardial infarction

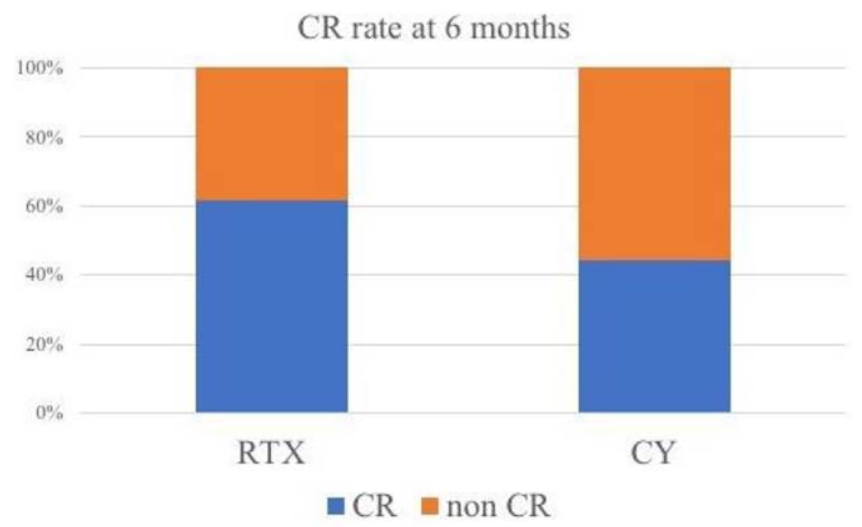

Figure 1. Complete remission rate at 6 month in the two groups. CR: complete remission, RTX: Rituximab, CY: Cyclophosphamide

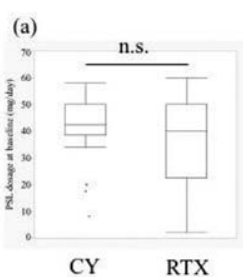

(b)

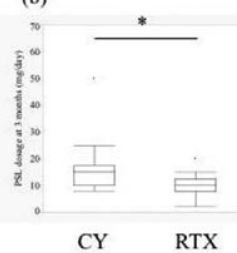

(c)

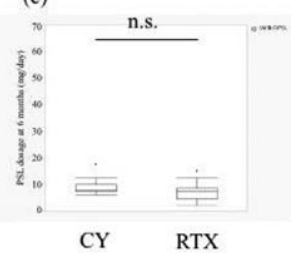

Figure 2. PSL dosage in two groups. (a) PSL dosage at baseline (b) PSL dosage at 3 months (c) PSL dosage at 6 months. PSL: prednisolone, CY: Cyclophosphamide, RTX: Rituximab, n.s. not significant, ${ }^{*} \mathrm{p}<0.05$

patients are characterized by the predominance of elderly, and the study about comparison of efficacy and safety between RTX and CY in elderly Japanese AAV patients are limited.

Objectives: To compare the efficacy and safety between RTX versus CY as remission induction therapy in Japanese AAV patients.

Methods: We analyzed 40 cases (20 cases received RTX and 20 cases received $\mathrm{CY}$ ) who received remission induction therapy in our hospital between January 2016 and August 2019. Clinical and laboratory variables at diagnosis, rates of complete remission(CR) at 6 months, defined as Birmingham Vasculitis Activity Score $(B V A S)=0$ and prednisone $7.5 \mathrm{mg} /$ day, AAV relapse at 12 months, and adverse effects were investigated.

Results: Of 40 patients, mean age was $73.5 \pm 9.6$ years $(6$ males and 34 females). Diagnosis of MPA and GPA were 30 cases and 10 cases, respectively. 37 cases (93\%) were positive for MPO-ANCA. Treatment regimen was determined by attending physician. Baseline characteristic of each group (RTX group and CY group) are shown in Table1. Baseline charactor, disease activity, organ involvement, and the proportion of patients with relapsing disease were similar in the two treatment groups. At 6 months, there was no difference of remission rate between two groups (RTX: CY $=62 \%: 44 \%, p=0.35$ ) (Figure 1). However, mean PSL dosage at 3 months was significantly lower in RTX group $(10.0 \pm 4.6 \mathrm{mg} /$ day) as compared to CY group $(15.8 \pm 9.5 \mathrm{mg} /$ day; $p=0.025)$ (Figure 2). At 12 months, 1 case in CY group and no case in RTX group had relapse. Adverse effects through 12 months are shown in Table 2. 8 infections $(30 \%)$ in CY group and 7 infections $(35 \%)$ occurred in RTX group $(p=0.64)$ respectively. 1 case in RTX group had died due to renal failure.

Conclusion: We indicated that PSL was tapered more rapidly in RTX group, although there was no difference of remission rate at 6 months and infection at 12 months between RTX and CY therapy. Therefore, remission induction therapy with RTX might be more safety for elderly Japanese AAV patients.

References:

[1] Little MA, Nightingale P, Verburgh CA, Hauser T, et al. Ann Rheum Dis 2010; 69:1036-1043.

Disclosure of Interests: None declared

DOI: 10.1136/annrheumdis-2020-eular.5377

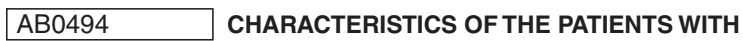 POLYARTERITIS NODOSA IN JAPAN}

M. Kawazoe ${ }^{1}$, T. Nanki $^{1}$, N. Hagino ${ }^{2}$, N. Iketani ${ }^{3}$, S. Ito ${ }^{4}$, M. Kodera ${ }^{5}$, N. Nakano ${ }^{6}$, M. Suzuki ${ }^{7}$, S. Y. Kaname ${ }^{3}$, M. Harigai ${ }^{8} .{ }^{1}$ Toho University, Division of Rheumatology, Department of Internal Medicine, School of Medicine, Faculty of Medicine, Tokyo, Japan; ${ }^{2}$ Division of Hematology and 